\title{
Pulsed entanglement measured by parametric amplifier assisted homodyne detection
}

\author{
Jiamin LI, ${ }^{1}$ (i) Yuhong LiU, ${ }^{1}$ (i) NAn Huo, ${ }^{1}$ Liang CUI, ${ }^{1}$ Chang \\ FENG, ${ }^{1}$ Z. Y. OU, ${ }^{1,2}$ AND XIAOYING LI ${ }^{1,{ }^{*}}$ \\ ${ }^{1}$ College of Precision Instrument and Opto-electronics Engineering, Tianjin University, Key Laboratory of \\ Optoelectronics Information Technology of Ministry of Education, Tianjin 300072, China \\ ${ }^{2}$ Department of Physics, Indiana University-Purdue University Indianapolis, Indianapolis, IN 46202, USA \\ *xiaoyingli@tju.edu.cn
}

\begin{abstract}
Balanced homodyne detection relies on a beam splitter to superpose the weak signal input and strong local oscillator. However, recent investigation shows that a high gain phase sensitive amplifier (PSA) can be viewed as homodyne detector, in which the strong pump of PSA serves as the local oscillator [1]. Here, we analyze a new method of measuring the continuous variable entanglement by assisting a balanced homodyne detector with the PSA and implement it experimentally. Before measuring quadrature amplitude with the balanced homodyne detectors, two entangled fields generated from a pulse pumped fiber optical parametric amplifier are simultaneously coupled into the PSA. We find that the normalized noise for both the difference and sum of the quadrature amplitudes of the two entangled fields fall below the shot noise limit by about $4.6 \mathrm{~dB}$, which is the record degree of entanglement measured in optical fiber systems. The experimental results illustrate that the advantages of the new measurement method include but not limit to tolerance to detection loss and characterizing entanglement with only one homodyne detector. The influence of mode-mismatching due to multi-mode property of entanglement on the measured noise reduction can also be greatly mitigated, indicating the new method is advantageous over the traditional measurement in multi-mode case.
\end{abstract}

(C) 2019 Optical Society of America under the terms of the OSA Open Access Publishing Agreement

\section{Introduction}

Quantum entanglement of continuous variables (CV) is an essential resource for quantum information science and quantum metrology. Comparing with its discrete variable counterparts, $\mathrm{CV}$ entanglement has the advantage of unconditional generation [2]. So far, quantum teleportation, quantum dense coding, quantum image, quantum logic gates, and quantum enhanced precision measurement have been experimentally realized [2-7]. Among these proof-of-principle experimental demonstrations, joint measurement realized by two sets of balanced homodyne detection (HD) is widely used for measuring the $\mathrm{CV}$ entanglements. However, the quantum fields measured by balanced HD, which relies on a 50/50 beam splitter to superpose the weak signal and the strong local oscillator (LO), is prone to losses, such as propagation loss, less-than-unit quantum efficiency of detectors and imperfect mode matching efficiency in HD [2-5,8]. So the quantum advantage is often hampered by losses in HD.

Furthermore, for the entanglement produced from a pulse-pumped traveling-wave optical parametric amplifier (OPA), the noise reduction due to the quantum correlation between two entangled fields is broadband [9-13], but the entanglement in pulsed state is usually in multitemporal modes [14,15], which are independent with each other. In this case, if the LO of each balanced HD is not perfectly matched with the eigen-modes of entangled fields, the extra thermal noise originated from the mode-mismatching between LOs and multi-mode entanglement might be much higher than vacuum noise. As a result, the observed quantum effect of pulsed entanglement is always smaller than what is anticipated [13]. 
Recently, our group had theoretically studied a new method of measuring CV quantum entanglement generated from OPA [16]. The new method is realized by assisting the balanced HDs with a phase sensitive amplifier (PSA). During the measurement, two entangled fields are simultaneously coupled into the PSA with high efficiency and the outputs of PSA are measured by either one or two balanced HDs. The idea of using PSA to characterize quantum noise reduction was first adopted by Flurin et al. to overcome the huge classical electronic noise in measuring the inseparability of entanglement of microwaves [17]. Recently, Shaked et al. extend this kind of homodyne detection to optical wavelength, and the squeezing generated from four-wave-mixing (FWM) in fiber was successfully measured by scanning the pump phase of PSA [1]. Indeed, a high gain PSA can be viewed as homodyne detector, in which the strong pump of PSA serves as the LO [1]. The noise reduction measured by high gain PSA have the advantage of detection loss tolerance in noise reduction measurements [1,18-21], however, the noise fluctuations of quantum states in [1] and [17] are determined by the power directly measured at the PSA output. So the high gain PSA can not be used to measure the noise reduction in the quadrature amplitudes of two entangled fields, $\hat{X}_{1} \mp \hat{X}_{2}$ and $\hat{Y}_{1} \pm \hat{Y}_{2}$, respectively, which is the key for implementing some quantum information processing tasks, such as quantum teleportation and quantum dense coding $[4,5,7,22,23]$. Our new method proposed in [16] inherit the advantages of the two kinds of HDs: the balanced HD and high gain PSA. The noise fluctuations of $\hat{X}_{1} \mp \hat{X}_{2}$ and $\hat{Y}_{1} \pm \hat{Y}_{2}$ of the entangled fields can be respectively and simultaneously measured by locking the PSA and by properly setting the LO phase of each balanced HD [16].

Comparing with the traditional method of measuring quantum correlation between two entangled fields with two sets of balanced HDs, the new method obviously has two merits: (i) the measurement is insensitive to the losses at detection; (ii) quantum entanglement between two fields of different types can be revealed by only measuring one field with a balanced HD, so that two balanced HDs respectively suitable for two different fields are not necessary. Therefore, the entangled state involved in the fields outside of the wavelength range of the mature detection technologies such as $2 \mu \mathrm{m}$ optical wave and atomic wave can be measured as long as the detection technology of the other field is available. Additionally, if two entangled fields are in multitemporal modes, the new method provides an additional advantage over the traditional method: the extra thermal noise induced by the mode-mismatching between multi-mode entanglement and $\mathrm{LO}$ can be diminished $[15,16]$.

In this paper, we experimentally verify the new method by measuring entanglement of twin beams generated by a pulse pumped fiber optical parametric amplifier (FOPA) [15]. We test and characterize the measurement under various conditions, including changing detection loss, and varying the pump powers of PSA and FOPA etc.. The results qualitatively agree with the theory predictions. Moreover, we find that both the noise variances for the difference and sum of the quadrature amplitudes of the signal and idler twin beams, $\left\langle\Delta^{2}\left(\hat{X}_{1}-\hat{X}_{2}\right)\right\rangle$ and $\left\langle\Delta^{2}\left(\hat{Y}_{1}+\hat{Y}_{2}\right)\right\rangle$, fall below the shot noise limit (SNL) by about $4.6 \mathrm{~dB}$, corresponding to the inseparability $I=0.68$ (lower than the classical limit of $I=2$ ), which is better than previous results for entanglement generated from optical fibers [13,24]. This is because the influences of detection loss and mode-mismatching due to multi-mode property on the measured degree of entanglement are greatly mitigated.

\section{Experiments}

Our experimental setup is shown in Fig. 1. The entangled signal and idler twin beams are generated from a phase insensitive FOPA consisting of two coarse wavelength division multiplexers (CWDM) and a $150 \mathrm{~m}$ dispersion shifted fiber (DSF1) which is submerged in liquid nitrogen (77 K) to suppress Raman scattering [25]. The central wavelengths for each channel of CWDM are 1531, 1551, and $1571 \mathrm{~nm}$, respectively. For each channel, the transmission efficiency at the central wavelength is $\sim 95 \%$, the isolation to the adjacent channels is $\sim 40 \mathrm{~dB}$, and the $1 \mathrm{~dB}$ 
bandwidth is about $16 \mathrm{~nm}$. CWDM1 is used to couple the pulsed pump and the reference light of seed injection into DSF1. The central wavelength of seed injection is centering at 1533.4 $\mathrm{nm}$. Since the zero dispersion wavelength of DSF1 is about $1548.5 \mathrm{~nm}$, the phase matching condition of FWM in DSF1 is satisfied for the pulsed pump (P1) centering at $1549 \mathrm{~nm}$. In the FWM process, the amplification of the signal photons at $\omega_{s}$ is accompanied by the generation of idler photons at $\omega_{i}$ due to the energy conservation condition $2 \omega_{p}=\omega_{s}+\omega_{i}$, where $\omega_{p}, \omega_{s}$ and $\omega_{i}$ are the frequencies of pump, signal and idler photons, respectively. The amplified signal and idler twin beams, separated by CWDM2, possesses the Einstein-Podolsky-Rosen (EPR) correlation $[3,26]$. The normalized noise correlation between two fields [3]

$$
R=\frac{\left\langle\Delta^{2}\left(\hat{X}_{1}-\hat{X}_{2}\right)\right\rangle}{\left\langle\Delta^{2}\left(\hat{X}_{1}-\hat{X}_{2}\right)\right\rangle_{S N L}}=\frac{\left\langle\Delta^{2}\left(\hat{Y}_{1}+\hat{Y}_{2}\right)\right\rangle}{\left\langle\Delta^{2}\left(\hat{Y}_{1}+\hat{Y}_{2}\right)\right\rangle_{S N L}}=(\mu-v)^{2}<1,
$$

and the inseparability criteria [27]

$$
I=\frac{\left\langle\Delta^{2}\left(\hat{X}_{1}-\hat{X}_{2}\right)\right\rangle}{\left\langle\Delta^{2}\left(\hat{X}_{1}-\hat{X}_{2}\right)\right\rangle_{S N L}}+\frac{\left\langle\Delta^{2}\left(\hat{Y}_{1}+\hat{Y}_{2}\right)\right\rangle}{\left\langle\Delta^{2}\left(\hat{Y}_{1}+\hat{Y}_{2}\right)\right\rangle_{S N L}}=2(\mu-v)^{2}<2
$$

are satisfied. Here, $\mu=\cosh (r)$ and $v=\sinh (r)$ with $\mu^{2}-v^{2}=1$ are the gain coefficients of FOPA, where the parameter $r$ denotes the nonlinear coupling coefficient determined by the phase matching and pump power of FOPA [13]. $\hat{X}_{1,2}=\hat{a}_{1,2}+\hat{a}_{1,2}^{\dagger}$ and $\hat{Y}_{1,2}=i\left(\hat{a}_{1,2}-\hat{a}_{1,2}^{\dagger}\right)$ are the conjugate quadrature amplitudes of the signal and idler fields where $\hat{a}_{1,2}^{\dagger}$ and $\hat{a}_{1,2}^{\dagger}$ are the creation and annihilation operators of the two optical fields. In Eqs. (1) and (2), $\left\langle\Delta^{2}\left(\hat{X}_{1}-\hat{X}_{2}\right)\right\rangle_{S N L}=2$ and $\left\langle\Delta^{2}\left(\hat{Y}_{1}+\hat{Y}_{2}\right)\right\rangle_{S N L}=2$ are the SNLs obtained by assuming the two fields are replaced with vacuum. We have previously characterized the $\mathrm{CV}$ entanglement by using the traditional method [13], but here we will measure the entanglement using the new method.

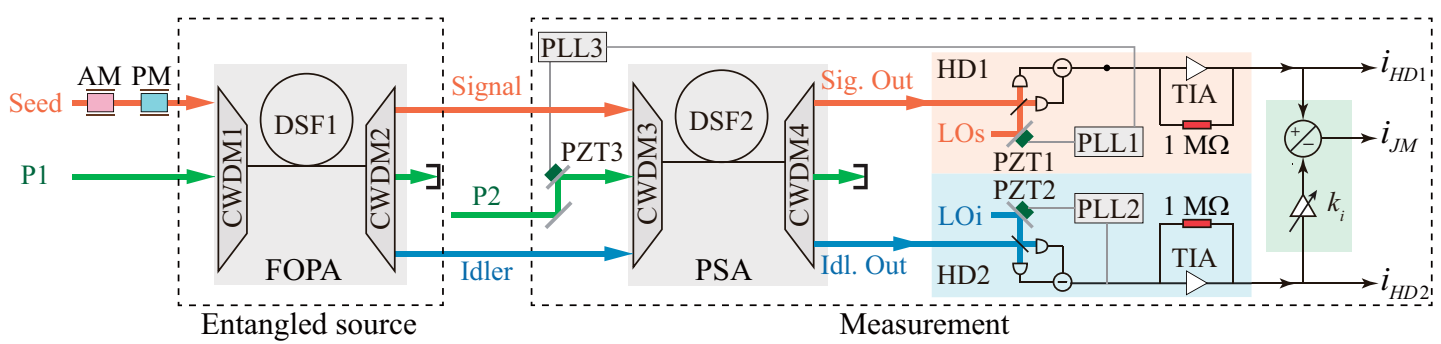

Fig. 1. Experimental setup of measuring entanglement generated from a fiber optical parametric amplifier (FOPA) by using the new method, realized by combining the PSA with balanced HDs. AM, amplitude modulator, PM, phase modulator; DSF, dispersion shifted fiber; CWDM, coarse wavelength division multiplexer; PSA, phase sensitive amplifier; P1, pump of FOPA; P2, pump of PSA; TIA, transimpedance amplifier; PZT, piezo mechanical transducer; HD, homodyne detector; LOs(i), local oscillator of HD1 (HD2).

Instead of directly coupling the entangled signal and idler fields into the balanced HD1 and HD2, respectively, we send the twin beams into the amplifier consisting of DSF2, CWDM3 and CWDM4, which are the same as the DSF1, CWDM1 and CWDM2 in FOPA, respectively. Since the signal and idler fields with non-zero intensities are simultaneously fed to the amplifier, the amplifier becomes phase sensitive and is referred to as PSA, which can transfer two inputs to two outputs without adding extra noise [28]. The central wavelength of the pump P2 is the same as that of P1. When the pulsed pump of PSA (P2) is properly phase locked to ensure the de-amplification operation condition, the noise at each output port of PSA is lower than the corresponding SNL because of destructive quantum interference effect originated from its two 


\section{Optics EXPRESS}

correlated inputs [29]. At the signal (idler) output of PSA, the quadrature amplitudes of $\hat{X}_{1(2)}^{\text {out }}$ and $\hat{Y}_{1(2)}^{\text {out }}$ are detected by HD1 (HD2) with the phase of LOs (LOi) locked at 0 and $\pi / 2$, respectively. In the measurement, the SNLs $\left\langle\Delta^{2} \hat{X}_{1(2)}^{\text {out }}\right\rangle_{S N L}$ and $\left\langle\Delta^{2} \hat{Y}_{1(2)}^{\text {out }}\right\rangle_{S N L}$ are obtained by replacing the two inputs of the PSA with vacuum. To obtaine the noise correlations of two entangled fields, the photo-currents of each balanced HD and the joint measurement of two HDs are respectively processed and analyzed by using the data acquisition (DAQ) system [22].

According to our analysis in [16], the noise fluctuation of the quadrature amplitudes at signal output of PSA, $\hat{X}_{1}^{\text {out }}$ and $\hat{Y}_{2}^{\text {out }}$, measured by individual HD1 are given by:

$$
\begin{aligned}
& \left\langle\Delta^{2} \hat{X}_{1}^{\text {out }}\right\rangle=G^{2}\left\langle\Delta^{2}\left(\hat{X}_{1}-\frac{g}{G} \hat{X}_{2}\right)\right\rangle, \\
& \left\langle\Delta^{2} \hat{Y}_{1}^{\text {out }}\right\rangle=G^{2}\left\langle\Delta^{2}\left(\hat{Y}_{1}+\frac{g}{G} \hat{Y}_{2}\right)\right\rangle,
\end{aligned}
$$

where $G$ and $g$ with $G^{2}-g^{2}=1$ are the gain coefficient of PSA. Using the corresponding SNLs

$$
\left\langle\Delta^{2} \hat{X}_{1}^{\text {out }}\right\rangle_{S N L}=\left\langle\Delta^{2} \hat{Y}_{1}^{\text {out }}\right\rangle_{S N L}=G^{2}+g^{2},
$$

we arrive at the normalized noises

$$
\begin{aligned}
& R_{1 X}=\frac{\left\langle\Delta^{2} \hat{X}_{1}^{\text {out }}\right\rangle}{\left\langle\Delta \hat{X}_{1}^{\text {out }}\right\rangle_{S N L}}=\frac{G^{2}\left\langle\Delta^{2}\left(\hat{X}_{1}-\frac{g}{G} \hat{X}_{2}\right)\right\rangle}{G^{2}+g^{2}} \\
& R_{1 Y}=\frac{\left\langle\Delta^{2} \hat{Y}_{1}^{\text {out }}\right\rangle}{\left\langle\Delta \hat{Y}_{1}^{\text {out }}\right\rangle_{S N L}}=\frac{G^{2}\left\langle\Delta^{2}\left(\hat{Y}_{1}+\frac{g}{G} \hat{Y}_{2}\right)\right\rangle}{G^{2}+g^{2}}
\end{aligned}
$$

For the case of $G, g \gg 1$, that is, $G \approx g$, they approach to

$$
\begin{aligned}
& R_{1 X} \approx \frac{\left\langle\Delta^{2}\left(\hat{X}_{1}-\hat{X}_{2}\right)\right\rangle}{\left\langle\Delta^{2}\left(\hat{X}_{1}-\hat{X}_{2}\right)\right\rangle_{S N L}}=(\mu-v)^{2}<1 \\
& R_{1 Y} \approx \frac{\left\langle\Delta^{2}\left(\hat{Y}_{1}+\hat{Y}_{2}\right)\right\rangle}{\left\langle\Delta^{2}\left(\hat{Y}_{1}+\hat{Y}_{2}\right)\right\rangle_{S N L}}=(\mu-v)^{2}<1
\end{aligned}
$$

Defining the coefficient $I_{H D 1}^{a m p}=R_{1 X}+R_{1 Y}$, it is straight forward to deduce that inseparability criteria $I_{H D 1}^{a m p} \approx I=\frac{\left\langle\Delta^{2}\left(\hat{X}_{1}-\hat{X}_{2}\right)\right\rangle}{\left\langle\Delta^{2}\left(\hat{X}_{1}-\hat{X}_{2}\right)\right\rangle_{S N L}}+\frac{\left\langle\Delta^{2}\left(\hat{Y}_{1}+\hat{Y}_{2}\right)\right\rangle}{\left\langle\Delta^{2}\left(\hat{Y}_{1}+\hat{Y}_{2}\right)\right\rangle_{S N L}}<2$ is satisfied for $G \approx g \gg 1$. Equation (6) indicates that the quantum correlation and inseparability of two entangled beams can be measured by placing a balanced HD at one output of the high gain PSA. This is because the function of coherently combining signal and idler fields in high gain PSA is equivalent to measuring the twin beam by performing the joint measurement of two sets balanced HDs [2]. Note that for HD2 at idler output port of PSA, the expressions of normalized noise $R_{2 X, 2 Y}$ and $I_{H D 2}^{a m p}=R_{2 X}+R_{2 Y}$ are similar to $R_{1 X, 1 Y}$ and $I_{H D 1}^{a m p}$, except that we need to switch $g$ and $G$ in Eqs. (3) and (5). When the condition $G \approx g \gg 1$ is satisfied, we have the normalized noise $R_{2 X, 2 Y}=R_{1 X, 1 Y}$ and the inseparability $I_{H D 2}^{a m p} \approx I_{H D 1}^{a m p} \approx I<2$. Moreover, it is worth pointing out that it is impossible to achieve $g / G$ or $G / g=1$ in a experiment system. Fortunately, our simulation result in [16] shows that as long as the ratio between the gain of PSA and FOPA, $G / \mu$, is high enough, the approximation in Eq. (6) holds.

We can also make joint measurement at the two outputs by analyzing the difference and sum of photo-currents of HD1 and HD2 with their LO phases respectively locking at 0 and $\pi / 2$. The 
noise fluctuations of jointly measured operators, $\hat{X}_{-}^{\text {out }}=\hat{X}_{1}^{\text {out }}-k \hat{X}_{2}^{\text {out }}$ and $\hat{Y}_{+}^{\text {out }}=\hat{Y}_{1}^{\text {out }}+k \hat{Y}_{2}^{\text {out }}$, are given by

$$
\begin{aligned}
& \left\langle\Delta^{2} \hat{X}_{-}^{\text {out }}\right\rangle=(G+k g)^{2}\left\langle\Delta^{2}\left(\hat{X}_{1}-\frac{g+k G}{G+k g} \hat{X}_{2}\right)\right| \\
& \left\langle\Delta^{2} \hat{Y}_{+}^{\text {out }}\right\rangle=(G+k g)^{2}\left\langle\Delta^{2}\left(\hat{Y}_{1}+\frac{g+k G}{G+k g} \hat{Y}_{2}\right)\right\rangle
\end{aligned}
$$

where $k$ is an adjustable parameter of electronic gain. Similarly, after normalizing with the corresponding SNLs of the joint measurement, we find the normalized noises of $\hat{X}_{-}^{\text {out }}$ and $\hat{Y}_{+}^{\text {out }}$

$$
\begin{aligned}
& R_{0 X}=\frac{\left\langle\Delta^{2} \hat{X}_{-}^{\text {out }}\right\rangle}{\left\langle\Delta^{2} \hat{X}_{-}^{\text {out }}\right\rangle_{S N L}}=\frac{\left\langle\Delta^{2}\left(\hat{X}_{1}-\hat{X}_{2}\right)\right\rangle}{\left\langle\Delta^{2}\left(\hat{X}_{1}-\hat{X}_{2}\right)\right\rangle_{S N L}}=(\mu-v)^{2} \\
& R_{0 Y}=\frac{\left\langle\Delta^{2} \hat{Y}_{+}^{\text {out }}\right\rangle}{\left\langle\Delta^{2} \hat{Y}_{+}^{\text {out }}\right\rangle_{S N L}}=\frac{\left\langle\Delta^{2}\left(\hat{Y}_{1}+\hat{Y}_{2}\right)\right\rangle}{\left\langle\Delta^{2}\left(\hat{Y}_{1}+\hat{Y}_{2}\right)\right\rangle_{S N L}}=(\mu-v)^{2}
\end{aligned}
$$

for $k=1$ are the same as $R$ in Eq. (1), and the inseparability criteria $I_{J M}^{a m p}=I=R_{0 X}+R_{0 Y}<2$ is always satisfied. Equation (8) shows that the normalized noise $R_{O X, O Y}$ are irrelevant to the gain of PSA. It is worth emphasizing that Eqs. (7) and (8) clearly illustrate the traditional method of measuring entanglement by using two sets of balanced HD is just a specific case of the new method under the condition that the gain of PSA is $G=1$ at which the pump of PSA is turning off and PSA function as a transmission medium of twin beams. Comparing with the traditional method, the advantage of measuring entanglement by using the high gain PSA assisted balanced $\mathrm{HD}$ is the tolerance to detection loss. When the detection loss of $\operatorname{HD}\left(L_{d}\right)$ is taken into account, we still have

$$
R_{j X, j Y} \approx R=(\mu-v)^{2}<1 \quad(j=0,1,2)
$$

if the gain of PSA is high enough so that the relations

$$
\left(1-L_{d}\right)(G+g)^{2} \gg 1 \text { and }\left(1-L_{d}\right) G^{2} \gg 1
$$

are respectively satisfied for joint measurement and individual measurement of HD1 or HD2 [16].

In our experiment, in order to properly lock the relative phase between the $\mathrm{LO}$ and detected field in each $\mathrm{HD}$, the seed injection is sequentially propagated through a phase modulator (PM) and an amplitude modulator (AM) with modulation frequencies of 0.3125 and $0.625 \mathrm{MHz}$, respectively. In this case, both the weakly modulated signals of the PM and AM are transferred to the signal and idler outputs of PSA, which can serve as the error signal for phase locking. The phase of each LO is locked by feeding the AC output of the HD1 or HD2 to the digital phase locking loops (PLL), and by loading the feedback signals of PLL1/PLL2 to the piezo-electric transducers, PZT1 and PZT2. In the meantime, to ensure the PSA is working at the de-amplification condition, the pump of PSA (P2) is modulated by an AM (not shown in Fig. 1) at a frequency of $1.5625 \mathrm{MHz}$, the modulation transferred to signal output is used as the error signal to add the feedback signal on the PZT3 via PLL3. More detailed information about the working procedure of the three PLLs is presented in $[13,22]$.

The seed, pumps and the local oscillators of HD1 and HD2 used in our experiment are created by first dispersing the $50 \mathrm{MHz}$ train of $150 \mathrm{fs}$ pulses centering at $1560 \mathrm{~nm}$ from a mode-locked fiber laser with a programmable filter (Waveshaper 4000A from Finisar) to obtain three beams with a wavelength separation of about $15 \mathrm{~nm}$. The procedure and method used for controlling the power and polarization of the four optical fields are described in more detail in [22] and [13]. In the experiment, the full width at half-maximum (FWHM) for the pumps P1 is $0.28 \mathrm{~nm}$, and the FWHM of P2 is adjusted to be $0.4 \mathrm{~nm}$ to efficiently couple the signal and idler twin beams into PSA. 
We first measure the normalized noise fluctuations of the two conjugate quadrature amplitudes, $R_{1 X(2 X)}$ and $R_{1 Y(2 Y)}$, at individual signal (idler) output of PSA by using the HD1 (HD2) with relative phase locking at 0 and $\pi / 2$. In the measurement, the power gain of FOPA is fixed at about 2.7. The transmission efficiency from each output of PSA to each HD is about $80 \%$. The quantum efficiency for each detector of HD is about $96 \%$. The measured values of $R_{1 X, 1 Y}$ and $R_{2 X, 2 Y}$ as a function of average power of pump P2, $P_{2 a}$, are shown in Fig. 2(a). Figure 2(b) plots the power gain of the PSA as a function of $P_{2 a}$. Here, the gains of FOPA and PSA (equivalent to $\mu^{2}$ and $G^{2}$ in Eqs. (1) and (3)), defined by the ratio between the powers of seed at output and input ports of the amplifier, are measured by blocking the pump P2 and P1, respectively. Obviously, the gain of PSA increases with $P_{2 a}$. The measurements show we have $R_{1 X}=R_{1 Y}$ and $R_{2 X}=R_{2 Y}$ because the noise of quadrature amplitude measured at each output of PSA does not change with the phase of local oscillators of HDs [23]. One sees that when $P_{2 a}$ is lower than 1 $\mathrm{mW}, R_{j X}$ and $R_{j Y}(j=1,2)$ are higher than the normalized SNL. In particular, when the pump P2 is blocked $\left(P_{2 a}=0\right)$, the values of $R_{1 X, 2 X}$ and $R_{1 Y, 2 Y}$ are the highest due to the thermal nature of individual signal (idler) field out of FOPA [15]. However, the normalized noises decrease with the increase of $P_{2 a}$. When $P_{2 a}$ is greater than $2 \mathrm{~mW}$, both $R_{j X}$ and $R_{j Y}$ are lower than SNL. For each set of data, the descending slope of normalized noise become very small for $P_{2 a}$ higher than $3 \mathrm{~mW}$. Moreover, the normalized noise level is about 0.34, $4.6 \mathrm{~dB}$ lower than SNL, for $P_{2 a}$ greater than $4 \mathrm{~mW}$, at which the corresponding the power gain of PSA is about 10 (see Fig. 2(b)). The results of $R_{1 X, 1 Y}$ and $R_{2 X, 2 Y}$ qualitatively agree with what is predicted by Eqs. (3) and (6). We notice that the normalized noise of quadrature amplitudes $R_{2 X, 2 Y}$ measured at idler out is slightly higher than $R_{1 X, 1 Y}$ at signal out. We think this might originate from the influence of Raman scattering (RS), since the intensity of RS measured in the idler field (centering at 1565 $\mathrm{nm}$ ) is higher than that in signal field (centering at $1533.4 \mathrm{~nm}$ ) [25].
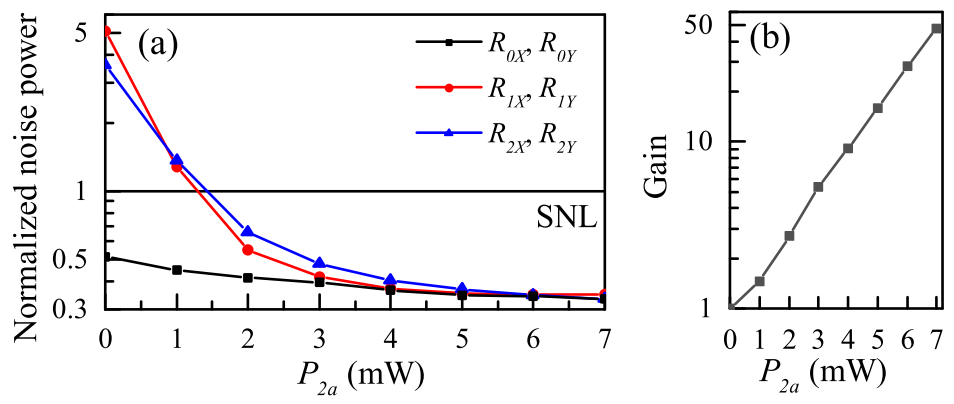

Fig. 2. (a) The normalized noise fluctuation the quadrature amplitudes, $R_{j X}$ and $R_{j Y}$, versus the average power of pump P2, $P_{2 a}$. The data for $j=1,2$ (triangles and circles) is obtained by the measurement of individual HD1 and HD2, while the data for $j=0$ (squares) is obtained by the joint measurement of two HDs. (b) The power gain of PSA as a function of $P_{2 a}$. The solid lines in the plots are only used for guiding eyes.

As a comparison, we also measure the normalized noise fluctuations $R_{0 X}$ and $R_{0 Y}$ from the joint measurement of the two HDs when the pump power $P_{2 a}$ is varied. We have $R_{0 X}=R_{0 Y}$, and the results (squares) are displayed in Fig. 2(a) as well. In contrast to the normalized noises measured by individual HD, we find $R_{0 X, 0 Y}<1$ always holds, which is irrelevant to $P_{2 a}$. However, $R_{0 X, O Y}$ also decreases with the increase of $P_{2 a}$. This is because the detection efficiency of each HD is not perfect, and the ability of detection-loss tolerance increases with the gain of PSA. Moreover, for a given pump power $P_{2 a}, R_{0 X, 0 Y}$ is always smaller than $R_{1 X, 1 Y}$ and $R_{2 X, 2 Y}$. The reasons are twofold. First, for the joint measurement, $R_{0 X, 0 Y}<1$ is always achievable; while $R_{1 X, 1 Y}<1$ and $R_{2 X, 2 Y}<1$ are realizable only for high gain PSA. Second, the influence of detection loss on jointly measured result of $R_{0 X, 0 Y}$ is less than that measured by individual $\operatorname{HDj}(j=1,2)$ (see Eq. (10)). It 
is worth noting that for $P_{2 a}$ greater than $6 \mathrm{~mW}, R_{0 X, 0 Y} \approx R_{1 X, 1 Y} \approx R_{2 X, 2 Y} \approx 0.34$ can be obtained for all the three cases in Fig. 2(a), which means the EPR entanglement measured by individual HD and joint measurement of two HDs is about the same for PSA with gain greater than 15 . The results illustrate that quantum entanglement between two different fields can be revealed by only measuring one field with a balanced HD (HD1 or HD2) in either signal or idler band if the gain of PSA is high enough, and two sets of balanced HDs are not necessary. Moreover, when the entanglement between two fields is characterized by using individual HD, the measurement results should also be insensitive to the loss induced by mode mis-match between the spectra of LO and measured field if the gain of PSA is high enough.

To further reveal that the ability of detection-loss tolerance increases with the gain of PSA, we perform joint measurement to measure $I_{J M}^{a m p}=R_{0 X}+R_{0 Y}$ by varying the detection loss of each HD when the gain of PSA is fixed at different levels. The total detection loss $\left(L_{d}\right)$ at each output port of PSA is the product of the detection loss of HD and the transmission loss and is changed by inserting an adjustable attenuator (ATT) in front of each HD. Figure 3 shows $I_{J M}^{a m p}$ as a function of the total detection loss when the gain of PSA is fixed at 1, 5 and 16, respectively. One sees the general trend of each set of data is the same: $I_{J M}^{a m p}$ always increases with the increase of detection loss. But the rising slope reduces with the increase of PSA gain. When the gain is 1, the rising slope of $I_{J M}^{a m p}$ is the highest. For a fixed value of loss, $I_{J M}^{a m p}$ decrease with the increase of gain. When total detection efficiency is in the range of about $60 \% \sim 80 \%$, the value of $I_{J M}^{a m p} \approx 0.68$ (4.6 dB lower than SNL) is achieved for PSA with gain of 16.

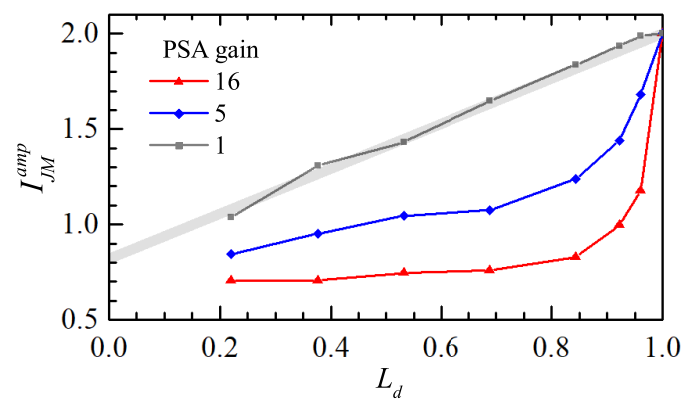

Fig. 3. The measured value of $I_{J M}^{a m p}$ as a function of total detection loss $\left(L_{d}\right)$ when the gain of PSA is 1,5 and 16 , respectively. The gray shadowed line is the linear fitting of the measured inseparability $I_{J M}^{a m p}$ with $G=1$ (circles) according to the function of $\left.I_{J M}^{a m p}\right|_{G=1}=I_{F O P A}^{\prime} \times\left(1-L_{d}\right)+2 L_{d}$, where $I_{F O P A}^{\prime}$ is the minimum inseparability by only correcting the detection loss. In the measurement, the gain of FOPA is fixed at 2.7.

In Fig. 3, the data (squares) obtained by blocking the pump P2 is equivalent to measuring the EPR correlation by using the traditional method. In this case, the gain of PSA is $G^{2}=1$, and the measured inseparability linearly depends on the detection loss due to the shot-noise coupled into detection system [16]. We fit this set of data by using the equation

$$
\left.I_{J M}^{a m p}\right|_{G=1}=I_{F O P A}^{\prime} \times\left(1-L_{d}\right)+2 L_{d}
$$

where the parameter $I_{F O P A}^{\prime}$ is the minimum value of inseparability achievable by completely compensating the loss due to non-ideal transmission and non-ideal quantum efficiency of photodetectors. According to the fitting (the gray shadowed line in Fig. 3), we find $I_{F O P A}^{\prime}=0.82$, which is worse than $I_{J M}^{a m p} \approx I_{H D j} \approx 0.68$ deduced from Fig. 2(a) for the case of $P_{2 a}$ greater than $5 \mathrm{~mW}$. The result indicates that there are other effects that are detrimental to entanglement measurement but cannot be attributed to the losses, and our new measurement method is not affected by these effects. Our analysis in the following shows that these effects are from multi-mode nature of the detected fields [16]. 
Since each filed of the entangled state generated by the pulse pumped FOPA is in multi-temporal modes [14,15], there exists an independent set of pairwise modes $\left\{\hat{A}_{j}, \hat{B}_{j}\right\}(j=1,2, \ldots)$ for the signal and idler fields that have the following input-output relation [13].

$$
\hat{A}_{j}^{\text {out }}=\mu_{j} \hat{A}_{j}^{\text {in }}+v_{j} \hat{B}_{j}^{\text {in } \dagger}, \hat{B}_{j}^{\text {out }}=\mu_{j} \hat{B}_{j}^{\text {in }}+v_{j} \hat{A}_{j}^{\text {in } \dagger},
$$

with $\hat{A}_{j}^{\dagger} \equiv \int d \omega_{s} \phi_{j}\left(\omega_{s}\right) \hat{a}_{1}^{\dagger}\left(\omega_{s}\right)$ and $\hat{B}_{j}^{\dagger} \equiv \int d \omega_{i} \psi_{j}\left(\omega_{i}\right) \hat{a}_{2}^{\dagger}\left(\omega_{i}\right)$ denoting the creation operators for $j$-th mode signal and idler fields, where the complex functions $\phi_{j}\left(\omega_{s}\right)$ and $\psi_{j}\left(\omega_{i}\right)$, satisfying the conjugate conditions, $\int d \omega_{s} \phi_{i}^{*}\left(\omega_{s}\right) \phi_{j}\left(\omega_{s}\right)=\delta_{i j}=\int d \omega_{i} \psi_{i}^{*}\left(\omega_{i}\right) \psi_{j}\left(\omega_{i}\right)$, represent the spectrum of signal and idler fields in the $j$-th order temporal modes $f_{j}(\tau)=\int d \omega_{s} \phi_{j}\left(\omega_{s}\right) e^{-i \omega_{s} \tau}$ and $h_{j}(\tau)=\int d \omega_{i} \psi_{j}\left(\omega_{i}\right) e^{-i \omega_{i} \tau}, \mu_{j}$ and $v_{j}$ with $\mu_{j}^{2}-v_{j}^{2}=1$ are the gain coefficient for $j-$ th mode. Since $\mu_{j}$ are different for different $j$, we can always arrange mode order so that $\mu_{1} \geqslant \mu_{2} \geqslant \mu_{3} \cdots$ $[14,15]$. For the pulsed pump with Gaussian shaped spectrum, the gain coefficient of the fundamental mode $\mu_{1}$ is the highest $[15,30]$.

In the multi-mode case, the strong local oscillators applied in HD1 and HD2 can be decomposed as

$$
A_{L O}(\omega)=\sum_{j} \xi_{j} \phi_{j}(\omega), \quad B_{L O}(\omega)=\sum_{j} \zeta_{j} \psi_{j}(\omega),
$$

where $\xi_{j} \equiv\left|\xi_{j}\right| e^{i \theta_{j}}=\int d \omega A_{L O}(\omega) \phi_{j}^{*}(\omega), \zeta_{j} \equiv\left|\zeta_{k}\right| e^{i \theta_{j}^{\prime}}=\int d \omega B_{L O}(\omega) \psi_{j}^{*}(\omega)$ with $\sum_{j}\left|\xi_{j}\right|^{2}=1=$ $\sum_{j}\left|\zeta_{j}\right|^{2}$ are the complex coefficient characterizing mode matching. $\theta_{j}, \theta_{j}^{\prime}$ are the LO phases for individual mode $j$. The theoretical analysis shows that the inseparability [16]

$$
I_{\text {multi }}=\sum_{j}\left[\left(\mu_{j}\left|\xi_{j}\right|-v_{j}\left|\zeta_{j}\right|\right)^{2}+\left(v_{j}\left|\xi_{j}\right|-\mu_{j}\left|\zeta_{j}\right|\right)^{2}\right]
$$

measured by the traditional method is an averaged value of inseparability of each mode pair, which are larger than that obtained by perfectly matching the local oscillators with fundamental modes $I_{1}=2\left(\mu_{1}-v_{1}\right)^{2}$ [15]. Moreover, if the mode matching coefficients for the two entangled fields $\zeta_{j}$ and $\xi_{j}$ are significantly different from each other, the measured value of $I_{m u l t i}$ in Eq. (14) might become higher than the SNL of 2 due to the thermal nature of individual signal or idler field. For example, assuming the entanglement is a super position of two pairs of temporal modes, if the mode matching coefficients between the detected fields and local oscillators are $\left|\xi_{1}\right|=0,\left|\xi_{2}\right|=1$ and $\left|\zeta_{1}\right|=1,\left|\zeta_{2}\right|=0$, the measured value of $I_{m u l t i}=\mu_{1}^{2}+v_{1}^{2}+\mu_{2}^{2}+v_{2}^{2}$ is higher than 2 , and the inseparability criterion can not be obtained.

When the entangled state is measured by the new method, the measured inseparability of PSA-assisted joint measurement can be written as [16]

$$
I_{J M}^{a m p} \approx \frac{2\left(\left|\xi_{1}\right|+\left|\zeta_{1}\right|\right)^{2} G_{1}^{2}\left(\mu_{1}-v_{1}\right)^{2}}{\left(\left|\xi_{1}\right|+\left|\zeta_{1}\right|\right)^{2} G_{1}^{2}}=2\left(\mu_{1}-v_{1}\right)^{2}
$$

for $g \rightarrow \infty$, which means that the difficulty encountered in directly measuring multi-mode entanglement with two balanced HDs can be solved.

To verify the merit of our new method on measuring the multi-mode entanglement, we jointly measure the normalized noise of quadrature amplitudes at signal and idler output fields and accordingly deduce the inseparability $I_{J M}^{a m p}$ when the power of PSA $P_{2 a}$ is set to either 0 or 7 $\mathrm{mW}$, corresponding to the power gain 1 and 48, respectively (see Fig. 2(b)). In each case, $I_{J M}^{a m p}$ is measured as a function of the average pump of FOPA. The gain of FOPA as a function of $P_{1 a}$ plotted in the inset of Fig. 4. The results of $I_{J M}^{a m p}$ versus $P_{1 a}$ for two cases are shown in the main plot of Fig. 4. Obviously, for a given power $P_{1 a}, I_{J M}^{a m p}$ measured for $P_{2 a}=7 \mathrm{~mW}$ are always better than those for $P_{2 a}=0$. We have known that the main reason is that the ability of detection-loss 
tolerance increases with the gain of PSA. However, even after correcting the data measured by the traditional method (for $P_{2 a}=0$ ) after taking the detection loss into account, the corrected data (circles) is still worse than the results for $P_{2 a}=7 \mathrm{~mW}$ (triangles). This is because the fields generated from pulse pumped FOPA are in multi-temporal modes but the signal and idler fields in the first order temporal modes $\phi_{1}\left(\omega_{s}\right)$ and $\varphi_{1}\left(\omega_{i}\right)$ become the dominating contribution at the high gain PSA [30].

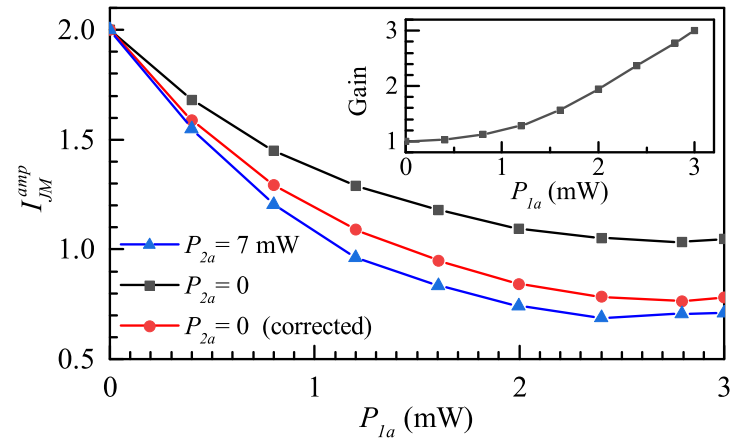

Fig. 4. Jointly measured inseparability $I_{J M}^{a m p}$ as a function average power of FOPA, $P_{1 a}$. The data represented by squares and triangles is respectively obtained for the case of $P_{2 a}=0$ and $P_{2 a}=7 \mathrm{~mW}$. In the measurements, the detection loss of each HD is about $L_{d}=0.22$. The solid circles are the corrected data for the case $P_{2 a}=0$ when the detection loss is taken into account. The inset shows the power gain of PSA as a function of average power $P_{1 a}$.

\section{Summary and discussion}

In conclusion, our experimental results illustrate that the new method realized by combining a high gain PSA with one or two balanced HDs has three advantages over the traditional method: tolerance to detection loss, resistance to temporal mode-mismatching, and only one balanced HD needed to characterize the entanglement between two different fields. It should be pointed out that the underlying physics in some recent experiments of using SU(1,1) nonlinear interferometer for quantum metrology is the same as the new method described here, that is, the noise for signal measurement is reduced due to entanglement correlation, and the noise reduction is tolerant to detection loss [22,31-33].

On the other hand, for the entanglement generated from a single pass optical parametric amplifier, particularly, by using ultra-fast mode locked laser as the pump, high speed detectors which can measure the broadband squeezing and resolve information encoded in individual pulse are highly desirable [34,35]. However, limited by the product between the gain of electronic amplifier and gain bandwidth, it is not trivial to develop such kind of fast detectors for quantum information processing. When PSA is used to assist the balanced HD in measuring the entanglement, the role of pump in the PSA is similar to that of the strong LO in balanced HD in the sense that the noise of entangled state and the level of SNL are amplified. Hence, the SNL of the new measurement method is lifted by the high gain PSA (see Eq. (4) and $[1,16]$ ). As a result, the requirement to the gain of the transimpedance amplifier (TIA) used to amplify photocurrent of HD (see Fig. 1) to get rid of the influence of dark currents on photodiodes is then relaxed. We expect to dramatically increase the response bandwidth of our new measurement system by reducing the gain of electronic amplifier of HD in the near future. 


\section{Optics EXPRESS}

\section{Funding}

National Natural Science Foundation of China (91836302,91736105,11527808); National Key

Research and Development Program of China (2016YFA0301403).

\section{References}

1. Y. Shaked, Y. Michael, R. Z. Vered, L. Bello, M. Rosenbluh, and A. Pe'er, "Lifting the bandwidth limit of optical homodyne measurement with broadband parametric amplification," Nat. Commun. 9(1), 609 (2018).

2. M. Reid, P. Drummond, W. Bowen, E. G. Cavalcanti, P. K. Lam, H. Bachor, U. L. Andersen, and G. Leuchs, "Colloquium: the Einstein-Podolsky-Rosen paradox: from concepts to applications," Rev. Mod. Phys. 81(4), 1727-1751 (2009).

3. Z. Y. Ou, S. F. Pereira, H. J. Kimble, and K. C. Peng, "Realization of the Einstein-Podolsky-Rosen paradox for continuous variables," Phys. Rev. Lett. 68(25), 3663-3666 (1992).

4. A. Furusawa, J. L. Sørensen, S. L. Braunstein, C. A. Fuchs, H. J. Kimble, and E. S. Polzik, "Unconditional quantum teleportation," Science 282(5389), 706-709 (1998).

5. X. Li, Q. Pan, J. Jing, J. Zhang, C. Xie, and K. Peng, "Quantum dense coding exploiting a bright Einstein-PodolskyRosen beam," Phys. Rev. Lett. 88(4), 047904 (2002).

6. S. L. Braunstein and P. van Loock, "Quantum information with continuous variables," Rev. Mod. Phys. 77(2), 513-577 (2005)

7. S. Steinlechner, J. Bauchrowitz, M. Meinders, H. Muller-Ebhardt, K. Danzmann, and R. Schnabel, "Quantum-dense metrology," Nat. Photonics 7(8), 626-630 (2013).

8. S. Cialdi, C. Porto, D. Cipriani, S. Olivares, and M. G. A. Paris, "Full quantum state reconstruction of symmetric two-mode squeezed thermal states via spectral homodyne detection and a state-balancing detector," Phys. Rev. A 93(4), 043805 (2016)

9. R. E. Slusher, P. Grangier, A. LaPorta, B. Yurke, and M. J. Potasek, "Pulsed squeezed light," Phys. Rev. Lett. 59(22), 2566-2569 (1987).

10. O. Aytür and P. Kumar, "Pulsed twin beams of light," Phys. Rev. Lett. 65(13), 1551-1554 (1990)

11. J. Wenger, R. Tualle-Brouri, and P. Grangier, "Pulsed homodyne measurements of femtosecond squeezed pulses generated by single-pass parametric deamplification,” Opt. Lett. 29(11), 1267-1269 (2004).

12. Y. Eto, T. Tajima, Y. Zhang, and T. Hirano, "Observation of quadrature squeezing in a $\chi^{(2)}$ nonlinear waveguide using a temporally shaped local oscillator pulse," Opt. Express 16(14), 10650-10657 (2008).

13. X. Guo, N. Liu, Y. Liu, X. Li, and Z. Y. Ou, "Generation of continuous variable quantum entanglement using a fiber optical parametric amplifier," Opt. Lett. 41(3), 653-656 (2016).

14. W. Wasilewski, A. I. Lvovsky, K. Banaszek, and C. Radzewicz, "Pulsed squeezed light: Simultaneous squeezing of multiple modes," Phys. Rev. A 73(6), 063819 (2006).

15. X. Guo, N. Liu, X. Li, and Z. Y. Ou, "Complete temporal mode analysis in pulse-pumped fiber-optical parametric amplifier for continuous variable entanglement generation," Opt. Express 23(23), 29369-83 (2015).

16. J. Li, Y. Liu, L. Cui, N. Huo, X. Li, and Z. Y. Ou, "Loss-tolerant measurement of continuous-variable quantum entanglement with the aid of a high gain parametric amplifier," arXiv 1808, 10258 (2018).

17. E. Flurin, N. Roch, F. Mallet, M. H. Devoret, and B. Huard, "Generating entangled microwave radiation over two transmission lines," Phys. Rev. Lett. 109(18), 183901 (2012).

18. C. Sparaciari, S. Olivares, and M. G. A. Paris, "Bounds to precision for quantum interferometry with gaussian states and operations," J. Opt. Soc. Am. B 32(7), 1354-1359 (2015).

19. C. Sparaciari, S. Olivares, and M. G. A. Paris, "Gaussian-state interferometry with passive and active elements," Phys. Rev. A 93(2), 023810 (2016).

20. B. Yurke, “Optical back-action-evading amplifiers,” J. Opt. Soc. Am. B 2(5), 732-738 (1985).

21. A. La Porta, R. E. Slusher, and B. Yurke, "Back-action evading measurements of an optical field using parametric down conversion," Phys. Rev. Lett. 62(1), 28-31 (1989).

22. Y. Liu, J. Li, L. Cui, N. Huo, S. M. Assad, X. Li, and Z. Y. Ou, "Loss-tolerant quantum dense metrology with SU(1,1) interferometer," Opt. Express 26(21), 27705 (2018)

23. J. Li, Y. Liu, L. Cui, N. Huo, S. M. Assad, X. Li, and Z. Y. Ou, "Joint measurement of multiple noncommuting parameters," Phys. Rev. A 97(5), 052127 (2018).

24. C. Silberhorn, P. K. Lam, O. Weiß, F. König, N. Korolkova, and G. Leuchs, "Generation of continuous variable Einstein-Podolsky-Rosen entanglement via the kerr nonlinearity in an optical fiber," Phys. Rev. Lett. 86(19), $4267-4270(2001)$

25. X. Guo, X. Li, N. Liu, L. Yang, and Z. Y. Ou, "An all-fiber source of pulsed twin beams for quantum communication," Appl. Phys. Lett. 101(26), 261111 (2012).

26. A. Einstein, B. Podolsky, and N. Rosen, "Can quantum-mechanical description of physical reality be considered complete?" Phys. Rev. 47(10), 777-780 (1935).

27. L.-M. Duan, G. Giedke, J. I. Cirac, and P. Zoller, "Inseparability criterion for continuous variable systems," Phys. Rev. Lett. 84(12), 2722-2725 (2000).

28. T. Li, B. E. Anderson, T. Horrom, B. L. Schmittberger, K. M. Jones, and P. D. Lett, "Improved measurement of two-mode quantum correlations using a phase-sensitive amplifier,” Opt. Express 25(18), 21301-21311 (2017). 


\section{Optics EXPRESS}

29. J. Kong, F. Hudelist, Z. Y. Ou, and W. Zhang, "Cancellation of internal quantum noise of an amplifier by quantum correlation,” Phys. Rev. Lett. 111(3), 033608 (2013).

30. N. Liu, Y. Liu, X. Guo, L. Yang, X. Li, and Z. Y. Ou, "Approaching single temporal mode operation in twin beams generated by pulse pumped high gain spontaneous four wave mixing," Opt. Express 24(2), 1096-1108 (2016).

31. F. Hudelist, J. Kong, C. Liu, J. Jing, Z. Y. Ou, and W. Zhang, "Quantum metrology with parametric amplifier-based photon correlation interferometers," Nat. Commun. 5(1), 3049 (2014).

32. M. Manceau, F. Khalili, and M. Chekhova, "Improving the phase super-sensitivity of squeezing-assisted interferometers by squeeze factor unbalancing," New J. Phys. 19(1), 013014 (2017).

33. B. E. Anderson, P. Gupta, B. L. Schmittberger, T. Horrom, C. Hermann-Avigliano, K. M. Jones, and P. D. Lett, "Phase sensing beyond the standard quantum limit with a variation on the SU(1,1) interferometer," Optica 4(7), 752-756 (2017).

34. R. Okubo, M. Hirano, Y. Zhang, and T. Hirano, "Pulse-resolved measurement of quadrature phase amplitudes of squeezed pulse trains at a repetition rate of $76 \mathrm{MHz}$," Opt. Lett. 33(13), 1458-1460 (2008).

35. Y.-M. Chi, B. Qi, W. Zhu, L. Qian, H.-K. Lo, S.-H. Youn, A. I. Lvovsky, and L. Tian, "A balanced homodyne detector for high-rate Gaussian-modulated coherent-state quantum key distribution," New J. Phys. 13(1), 013003 (2011). 